\title{
The model of complex structure of atomic nucleus and living body
}

\author{
Rongwu Liu* \\ Department of physics, Qufu Normal University, China
}

\begin{abstract}
Particle model has been challenged by three fields of the nature: The strong interaction and the weak interaction taking place in atomic nucleus, the state of black hole and the state of universe before Big Bang, the special life phenomena of human body (including parapsychological phenomena and paraphysiological phenomena). This article proposes a new mechanism of strong interaction and weak interaction, as well as a new structure model of matter (including atomic nucleus and living body).
\end{abstract}

\section{Introduction}

For a long time, the points of view about living body held by academic world have been divided into two kinds: one kind is reductionism, the other kind is vitalism. Reductionism believes that, living body, however complicated, all take particles as the basis of material structure ultimately, a variety of life phenomena are all different manifestations of the motion behaviors of particles, such as the function of biomacromolecule, it contributes to the comprehension of the motion behaviors of electrons and atomic nuclei [1-3]. Vitalism believes that, some phenomena of living body cannot be explained by particle view of matter, they follow the other set of natural laws, so there exists a kind of vital matter (or vital energy) in living body, some functions of living body are different manifestations of the same vital matter [4].

Natural world consists of biological world and non-biological world. Non-biological world has been studied very well by particle model, but there are still several problems that are not resolved, such as the mechanism of strong interaction and weak interaction, the state of black hole and the state of universe before Big Bang. This indicates that, there exist some areas that particle model isn't applicable in nonbiological world. For biological world, a lot of biological rules have been discovered in terms of particle model, but there are still some phenomena taking place inside and outside living body, that cannot be understood by particle model however [5]. This indicates that, there exist some phenomena that particle model cannot explain in biological world.

Based on this situation, this article proposes a new structure model of matter, which consists of the complex structure model of fundamental body, the atom-like structure model of hadron, the molecule-like structure model of atomic nucleus, the complex structure model of bio macromolecular backbone, the complex structure model of living body, the complex structure model of intelligent system of human body, and end up with the symmetry principle of particle motion system of non-living body and volume field motion system of living body.

\section{A new mechanism about strong interaction and weak interaction}

\section{Particle model versus volume field model}

Conventional physics are established on the basis of particle model. The particle model can be outlined as follows: Particle is a form of material existence in point space, it takes displacement motion in the form of continuous motion, particles have interactions (gravitational interaction, electromagnetic interaction, weak interaction, and strong interaction) between them by exchanging intermediate particles (graviton, photon, intermediate vector bosons $\mathrm{w}^{ \pm}$and $\mathrm{z}^{0}$, and gluon). The system in which particles take continuous displacement motion is referred to as particle motion system. This article suggests that, strong interaction and weak interaction don't follow particle model, they follow volume field model. The volume field model can be outlined as follows: Volume field is a form of material existence in plane space, it takes volume-changing motion (or volume motion for short) in the form of noncontinuous motion (or pulsation), volume fields have strong interaction or weak interaction between them by overlapping their volume fields. The system in which volume fields take non-continuous volume motion is referred to as volume field motion system.

\section{The characteristics of strong interaction and weak interaction}

There are five distinct characteristics about strong interaction and

Correspondence to: Rongwu Liu, Department of Physics, Qufu Normal University, China, Tel: (626) 371 5362; E-mail: rwliu65@gmail.com

Key words: living body, biomacromolecule, biomacromolecular backbone, biomacromolecular side chain radical, complex structure, fundamental body, fundamental matter, fundamental particle, fundamental volume field, particle-like quark, volume-field-like quark, volume-field-like neutrino, atomic nucleus, hadron, hadronic nucleus, extranuclear quarks, particle model, volume field model, particle motion system, volume field motion system, strong interaction, weak interaction, overlap force, plane space, volume-changing motion (or volume motion), non-continuous motion (or pulsation)

Received: July 29, 2016; Accepted: August 15, 2016; Published: August 20, 2016 
weak interaction. Short-range force: unlike gravitational interaction and electromagnetic interaction, strong interaction and weak interaction is a kind of short-range force which is limited within the scope of atomic nucleus [6], as if hadrons or quarks are held in a "bag" [7]. Overlap force: unlike gravitational interaction and electromagnetic interaction, strong interaction and weak interaction is a kind of overlap force, instead of exchange force. Saturation force: unlike gravitational interaction and electromagnetic interaction, strong interaction and weak interaction is a kind of saturation force that one particle participating in interaction cannot have action with the third particle [8]. Non-central force: unlike gravitational interaction and electromagnetic interaction, strong interaction and weak interaction is a kind of non-central force which cannot be expressed as a force along the line connecting the centers of two particles $[9,10]$. Non-attenuate force: unlike gravitational interaction and electromagnetic interaction, strong interaction and weak interaction is a kind of non-attenuate force which maintains constant within the force range $[11,12]$.

\section{The complex structure model of atomic nucleus}

The complex structure model of fundamental body Fundamental body and fundamental matter: Are there basic materials in the nature? Does electron or quark have inner structure? What are the components of electron or quark? For a long time, the above three questions have been the research frontiers of particle physics. In chemistry, atom is considered as the basic unit which cannot be divided any longer, even though it is composed of atomic nucleus and extra nuclear electrons. What is the smallest unit which cannot be divided any longer in physics? This article suggests that: Fundamental body is the smallest unit which cannot be divided any longer in physics, such as quark, it is further composed of different kinds of fundamental matter which are compatible with each other. Fundamental matter is the most basic material which constitutes the natural world. There are four kinds of fundamental matter in the nature, they are mass, electricity, flavor, and color, respectively. Experiments indicate that, fundamental matter has the attribute of interaction between each other, but only the same kinds of fundamental matter can interact between them. There exist four kinds of fundamental interactions between fundamental bodies in the nature, which include gravitational interaction, electromagnetic interaction, strong interaction, and weak interaction. These four fundamental interaction forces are generated by the interactions of the four kinds of fundamental matter-mass, electricity, color, and flavor, respectively.

\section{Fundamental particle and fundamental volume field}

In particle model, matter exists in the form of particle, any object is considered as composing of different level of particles. Volume field model suggests that: The fundamental matter constituting fundamental body exists in two different forms, fundamental matter mass and electricity exist in the form of particle, fundamental matter flavor and color exist in the form of volume field. The part of mass and electricity existing in the form of particle in fundamental body is called fundamental particle, the part of flavor and color existing in the form of volume field in fundamental body is called fundamental volume field. Fundamental particle lies in the interior of fundamental volume field, forms the "nucleus" of fundamental body. So, fundamental body is a kind of complex structure of fundamental particle and fundamental volume field (Figure 1).

\section{The predictions of dark matter particle, volume-field- like quark, and volume-field-like neutrino}

\author{
The combination principle of the least intensity of \\ fundamental body
}

How does fundamental matter constitute fundamental body? In terms of different number of fundamental matter which constitutes fundamental body, fundamental body is divided into four classes that includes one-in-one (or called single), two-in-one, three-in-one, and four-in-one. The one-in-one consists of one kind of fundamental matter, the two-in-one consists of two kinds of fundamental matter, the three-in-one consists of three kinds of fundamental matter, and the four-in-one consists of four kinds of fundamental matter. In fundamental body, the combination of fundamental matter is not arbitrary, but constructed on the basis of the "combination principle of the least intensity". What is called the "combination principle of the least intensity" means that, the sum of interaction intensities of the fundamental matter constituting fundamental body is the least. According to this principle, one-in-one consists only of fundamental matter mass, because among four kinds of fundamental matter, the interaction intensity between masses is the least; two-in-one consists only of fundamental matter mass and flavor, because among four kinds of fundamental matter, the sum of the interaction intensities between masses and between flavors is the least, such as neutrino; three-inone consists only of fundamental matter mass, flavor, and electricity, because among four kinds of fundamental matter, the sum of the interaction intensities between masses, between flavors, and between electricities is the least, such as electron; four-in-one consists of all kinds of fundamental matter mass, flavor, electricity, and color, such as quark (Table 1).

From Table 1 we find that, there exists fundamental body one-inone which only consists of fundamental matter mass. So far, the onein-one that only participates in gravitational interaction hasn't been found experimentally. This article suggests that, the fundamental body one-in-one might be the dark matter particle which forms the dark matter of universe.

\section{The self-motion state of fundamental volume field}

In particle model, the motion state of a particle is relative to object

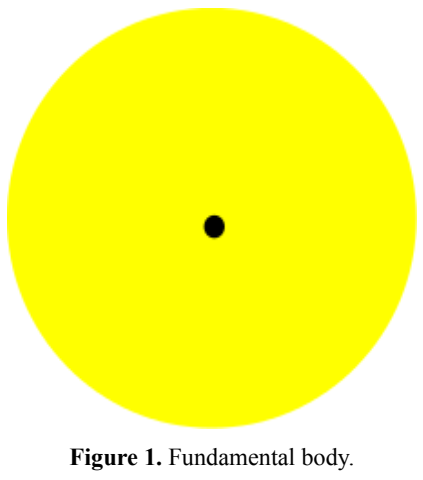

Table 1. The categories of fundamental body.

\begin{tabular}{|l|c|c|c|c|}
\hline & mass & flavor & electricity & color \\
\hline four-in-one (e.g. quark) & $\mathrm{x}$ & $\mathrm{x}$ & $\mathrm{x}$ & $\mathrm{x}$ \\
\hline three-in-one (e.g. electron) & $\mathrm{x}$ & $\mathrm{x}$ & $\mathrm{x}$ & \\
\hline two-in-one (e.g. neutrino) & $\mathrm{x}$ & $\mathrm{x}$ & & \\
\hline one-in-one (or single) & $\mathrm{x}$ & & & \\
\hline
\end{tabular}


of reference, so the motion and rest of a particle are relative. But in volume field model, the volume motion of volume field is absolute. When volume field takes volume motion (volume-expanding motion or volume-contracting motion), no matter how to choose object of reference, the volume (or density) of volume field makes change with time; when volume field is at rest (doesn't take volume motion), no matter how to choose object of reference, the volume (or density) of volume field keeps unchanged with time. Therefore, the state of volume motion of volume field depends on whether the volume (or density) of volume field changes or not with time. If the volume (or density) of a volume field changes over a period of time, then the volume field is in the state of absolute motion, at this time the volume field moves in the way of pulsation; if the volume (or density) of a volume field doesn't change over a period of time, then the volume field is in the state of absolute rest, even if the volume field takes displacement motion with particle, at this time the volume field doesn't take volume motion.

The fundamental volume field in fundamental body also has two states of absolute rest and absolute motion in isolation, we call them the self-motion states of fundamental volume field. The fundamental volume field whose self-motion state is in the static state is called static fundamental volume field, the corresponding color field and flavor field are called static fundamental color field and static fundamental flavor field, respectively. The fundamental volume field whose selfmotion state is in the moving state (or pulsating state) is called moving fundamental volume field, the corresponding color field and flavor field are called moving fundamental color field and moving fundamental flavor field, respectively. Like fundamental particle which has the particle properties of mass, charge, and spin etc, in volume field model fundamental volume field also has the volume field properties of flavor, color, and self-motion etc.

According to different self-motion states of fundamental volume fields, fundamental bodies are divided into two classes: One class is the fundamental bodies whose self-motion states of fundamental volume fields carried by the fundamental bodies are in the static states, or called the fundamental bodies carrying static fundamental volume fields. For this kind of fundamental body, the fundamental volume field carried by fundamental body is in the state of rest in isolation, that is, the volume (or density) of fundamental volume field doesn't vary with time when it exists alone (only when two static fundamental volume fields have interaction by means of overlap with each other, the overlapping volume fields are in the state of motion). We refer to this kind of fundamental body as particle-like fundamental body (Figure 2 left). Since the fundamental particle in particle-like fundamental body is in the relative moving state, and yet the fundamental volume field which coexists with fundamental particle is in the absolute static state, particle-like fundamental body behaves mainly as particle properties, it has the particle properties of mass, charge, spin, and average lifetime etc. So far, the fundamental bodies found experimentally are probably all particle-like fundamental bodies, particle model just takes this kind of fundamental body as research object. The other class is the fundamental bodies whose self-motion states of fundamental volume
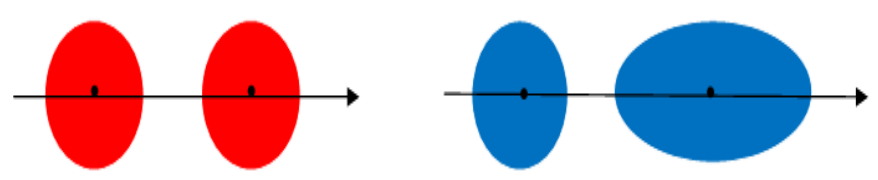

Figure 2. Particle-like fundamental body and volume-field-like fundamental body. fields carried by the fundamental bodies are in the moving states, or called the fundamental bodies carrying moving fundamental volume fields. For this kind of fundamental body, the volume field carried by fundamental body is in the state of absolute motion in isolation, that is, the volume or density of fundamental volume field varies with time even if it exists alone. We refer to this kind of fundamental body as volume-field-like fundamental body (Figure 2 right). Since the fundamental volume field in volume-field-like fundamental body is in the absolute moving state, and yet the fundamental particle which coexists with fundamental volume field is in the follow-up state, volume-field-like fundamental body behaves mainly as volume field properties, it has the volume field properties of flavor, color, and selfmotion etc. Since fundamental volume field takes volume motion in the form of non-continuous motion, it's not easy for volume-field-like fundamental body to be observed on the experiment designed under the guidance of particle model.

\section{The condition that volume-field-like fundamental body exists}

Does each kind of fundamental body (such as, one-in-one, twoin-one, three-in-one, and four-in-one) exist both in the form of particle-like fundamental body and in the form of volume-field-like fundamental body in the nature? In another word, whether or not each kind of particle-like fundamental body corresponds to a kind of volumefield-like fundamental body, respectively. For example, whether or not there exists volume-field-like electron which corresponds to particlelike electron.

According to the definition of volume-field-like fundamental body, the fundamental volume fields in volume-field-like fundamental body are in the state of active motion, and the fundamental particles which coexist with fundamental volume fields in volume-field-like fundamental body are in the state of passive motion. This situation requires that, in general material environment, the action intensities of the fundamental volume fields of fundamental body with surrounding must be greater than the action intensities of the fundamental particles of fundamental body with surrounding. Only in this way, it can ensure that the fundamental volume fields in fundamental body are in the state of active motion. Therefore we may suppose that, the condition that volume-field-like fundamental body exists is that: the sum of the action intensities of the fundamental volume fields in fundamental body is greater than the sum of the action intensities of the fundamental particles in fundamental body.

According to this assumption, one-in-one is a kind of particlelike fundamental body, this is because, one-in-one is only composed of fundamental matter mass, and fundamental matter mass exists in the form of particle; two-in-one exists both in the form of particle-like fundamental body and in the form of volume-field-like fundamental body, this is because, in two-in-one the action intensity of fundamental volume field flavor is greater than the action intensity of fundamental particle mass, such as neutrino, which should exist in two forms of particle-like neutrino and volume-field-like neutrino; three-inone only exists in the form of particle-like fundamental body, this is because, in three-in-one the action intensity of the fundamental volume field flavor is less than the sum of the action intensities of fundamental particles mass and electricity, such as electron, which might exist only in the form of particle-like electron; four-in-one exists both in the form of particle-like fundamental body and in the form of volume-field-like fundamental body, this is because, in four-in-one the sum of the action intensities of fundamental volume fields flavor and color is greater than the sum of the action intensities of fundamental particles mass 
and electricity, such as quark, which should also exist in two forms of particle-like quark and volume-field-like quark. In conclusion, one-inone and three-in-one are all particle-like fundamental bodies, two-inone and four-in-one exist both in the form of particle-like fundamental body and in the form of volume-field-like fundamental body.

\section{The atom-like structure model of hadron}

In atom model, atom is composed of the positively charged atomic nucleus which is in the state of relative rest and the negatively charged electrons which are in the state of relative motion outside atomic nucleus. The atomic nucleus is further composed of positively charged protons and uncharged neutrons, the extra nuclear electrons move in different shells in terms of different energy levels. The electrified bodies in atom interact between them by means of emitting and exchanging electromagnetic field quantum-photons. Similar to atom model, in volume field model hadron has atom-like structure, it is composed of hadronic nucleus and extra nuclear quarks. The hadronic nucleus (that is, the "hadron" in quark model $[13,14]$ ) is further composed of particle-like quarks which carry static fundamental volume fields with different monochromes-red, yellow, and blue (or red, blue, and green) $[15,16]$, the quarks interact between them by means of overlapping static fundamental color fields, hadronic nucleus is colorless as a whole; while the extra nuclear quarks are all volume-field-like quarks which carry moving fundamental volume fields with the same compound color-white, the quarks interact between them by means of overlapping moving fundamental color fields, as a result, hadron is white as a whole. In order to match the description about mass and charge of hadron in particle model, we must assume that the volumefield-like quark outside hadronic nucleus carry a little bit mass (like neutrino) and electrically neutral as a whole. Since extra nuclear quarks interact between them by overlapping moving fundamental color fields carried by the quarks, the extra nuclear quarks of hadron take volume motion under strong interaction, therefore forms extra nuclear moving color field current of hadron (or called the extra nuclear current of volume-field-like quarks of hadron) (Figure 3).

\section{The molecule-like structure model of atomic nucleus}

In the structure model of molecule, molecule is composed of atoms, the atoms interact between them by means of shifting the valence electrons (forming ionic bond) or sharing the electrons (forming covalent bond) of outer shell of atom. This interaction force is referred to as chemical bond, it is a kind of electrical attractive force in essence. The outer electrons of atoms in molecule take collective motion inside molecule, therefore forms molecular electric current in molecule. Similar to molecular model, in volume field model atomic nucleus is composed of hadrons, the hadrons interact between them by

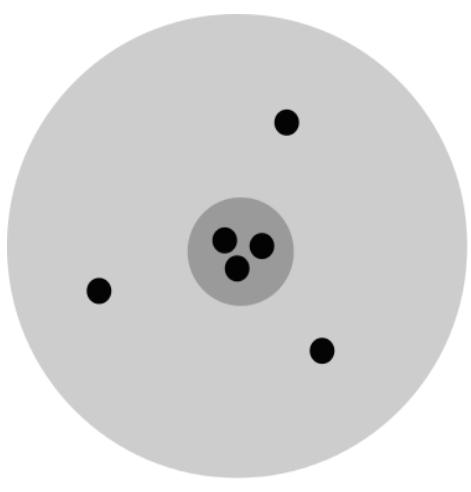

Figure 3. The atom-like structure of hadron. means of overlapping extra nuclear moving color fields of hadrons. The overlapping moving color fields between hadronic nuclei take volume motion under strong interaction, therefore forms moving color field current of atomic nucleus (or called the current of volume-field-like quarks of atomic nucleus, similar to the molecular electric current in molecule). Since the volume of volume field is limited, atomic nucleus is like a "bag" which is full of volume fields, as well as the particles compatible with volume fields (Figure 4).

\section{The complex structure model of bio macromolecular backbone}

The complex structure models of hadron and atomic nucleus reveal that, there exists a volume field motion system in microscopic field of natural world. Is there a volume field motion system in macroscopic field of natural world?

In particle model, particle motion system is divided into microscopic motion system of particle and macroscopic motion system of particle. The microscopic motion system of particle exists in the structure levels below molecule (including molecule), such as atomic system and molecular system; the macroscopic motion system of particle exists in the structure levels above molecule, such as molecular polymer system (including metal conductor and semiconductor), solar system etc.We may suppose that, like particle motion system, in volume field model volume field motion system is also divided into microscopic motion system of volume field and macroscopic motion system of volume field. The microscopic motion system of volume field exists in the structure levels below atomic nucleus (including atomic nucleus), such as hadronic system and atomic nuclear system; the macroscopic motion system of volume field exists in the structure levels above atomic nucleus. Then, in what kind of object does macroscopic motion system of volume field exist in the nature? No sign indicates that there exists volume field motion system in macroscopic field of non-living body. What about living body? There are a lot of evidences (such as special life phenomena-parapsychological phenomena and Para physiological phenomena-taking place around or within human body) which indicate that there exists macroscopic motion system of volume field in living body.

The studies of Molecular Biology and Biochemistry indicate that, the essential difference between living body and non-living body is that, there exist bio macromolecules which reflect life vitality in living body, to say specifically, they are protein and nucleic acid. These two kinds of bio macromolecules are generally known as living matter. Bio macromolecule is a kind of chain macromolecule which is composed of various kinds of amino acids or nucleotides connected by chemical bonds. Bio macromolecule consists of two parts of bio macromolecular

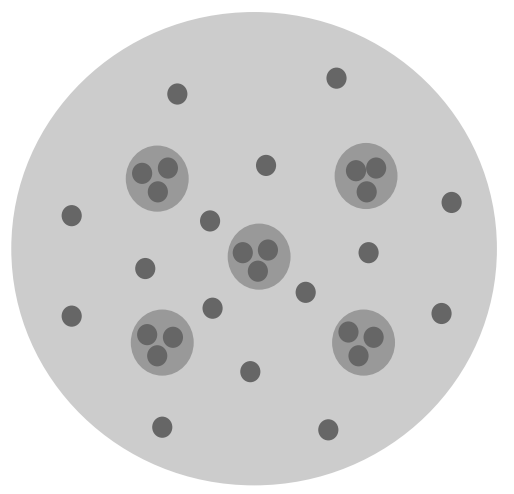

Figure 4. The molecule-like structure of atomic nucleus. 
backbone (or main chain) and bio macromolecular side chain radical (or side chain group). The bio macromolecular backbones are all neat crystal structures which are constructed in a specific geometric pattern, while the side chain radicals are all general loose material structures.

Is there macroscopic volume field in bio macromolecule? We may propose that, as there exist free electrons in metal conductor and semiconductor, there exist free volume-field-like quarks which carry moving fundamental volume fields in the space around atomic nuclei of bio macromolecular backbone, they have strong interaction between them by means of overlap with each other, the overlapping volume fields take volume motion. Thus, bio macromolecule is a complex motion system which consists of particle motion system and volume field motion system. The former mainly exists in the bio macromolecular side chain radical (regard the atomic nuclei of bio macromolecular side chain radical as particles), the latter mainly exists in the bio macromolecular backbone (except the electrons of bio macromolecular backbone and regard the hadronic nuclei of bio macromolecular backbone as volume fields) (Figure 5).

\section{The complex structure model of living body}

\section{The assumption of the distribution of one-in-one (or single)}

According to the combination principle of the least intensity of fundamental body, there exists fundamental body one-in-one (or single) which only consists of fundamental matter mass. We may suppose that, singles exist around all particles (including macroscopic objects) which possess rest masses, gravitational interaction takes place between singles, between single and the other particle. According to this assumption, in the system of molecular polymer consisting of atomic nuclei and electrons, it is filled with a cloud of "single gas" which is composed of singles in the space around atomic nuclei and electrons; in the system of heavenly body consisting of molecular polymers, it is also filled with a cloud of "single gas" in the space around molecular polymers; in the system of galaxy consisting of heavenly bodies, it is also filled with a cloud of "single gas" in the space around heavenly bodies; even in the system of universe consisting of galaxies, it is also filled with a cloud of "single gas" in the space around galaxies (Figure 6). This discussion also applies to living body. Since fundamental body one-in-one only carries mass and distributes everywhere, it is hard to detect it experimentally, probably this is the reason why we haven't found this kind of particle so far. The assumption of the distribution of fundamental body one-in-one in non-living body can account for the cause of formation of the dark matter of the universe.

\section{The assumption of the distribution of volume-field-like neutrino}

We may also suppose that, volume-field-like neutrinos exist

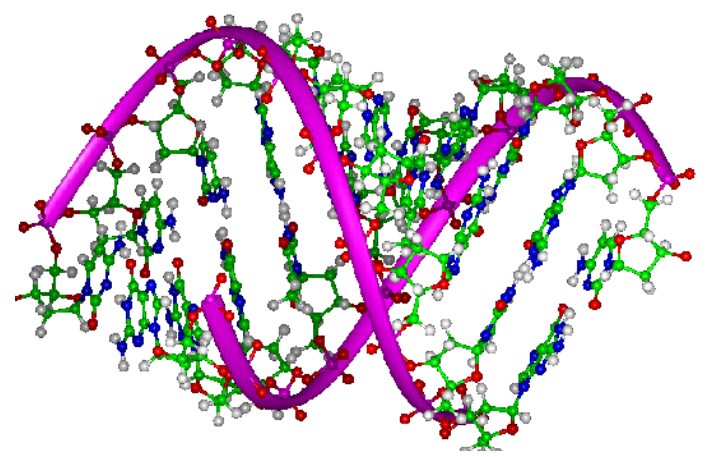

Figure 5. The assumption of free quarks in biomacromolecular backbone.

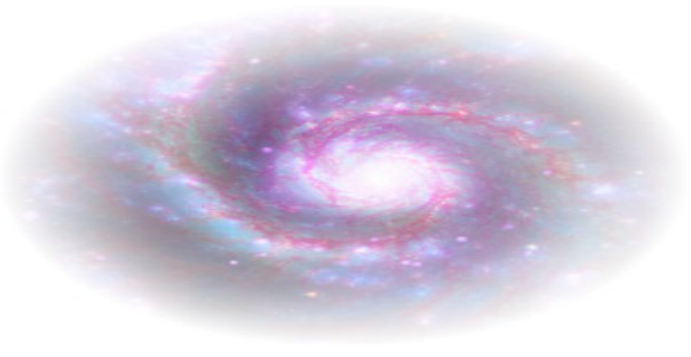

Figure 6. The galaxy filled with "single gas".

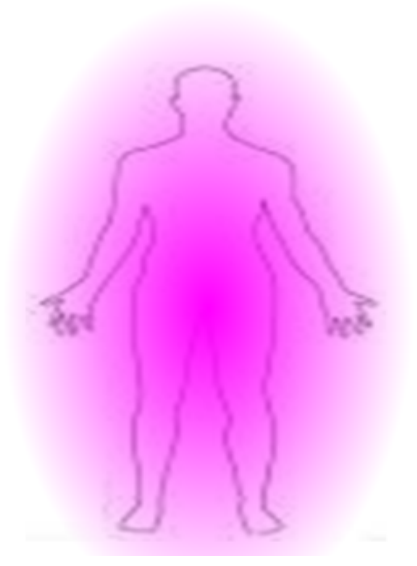

Figure 7. The human body filled with volume-field-like neutrinos.

around all volume fields which carry flavors, weak interaction takes place between volume-field-like neutrinos, between volume-field-like neutrino and the other volume field. According to this assumption, in the system of biomacromolecular backbone consisting of hadronic nuclei and volume-field-like quarks, it is filled with a cloud of "neutrino chi field" (or called weak chi field) which is composed of volume-fieldlike neutrinos in the space around hadronic nuclei and volume-fieldlike quarks; in the system of biomacromolecular polymer (refers to the part of biomacromolecular backbones, the same below) consisting of biomacromolecular backbones, it is filled with a cloud of "neutrino chi field" in the space around biomacromolecular backbones; in the system of human body consisting of biomacromolecular polymers, it is filled with a cloud of "neutrino chi field" in the space around biomacromolecular polymers; even in the system of living world consisting of living bodies, it is filled with a cloud of "neutrino chi field" in the space around living bodies (Figure 7). The assumption of the distribution of fundamental body volume-field-like neutrino in living body can account for the essence of "chi of viscera" in the theory of traditional Chinese medical.

\section{The assumption of soul system}

Through the above discussion we know that, any living body is a kind of complex structure of particle motion system and volume field motion system, the two motion systems function separately, along together form biological function of living body. For intelligent living body, intelligent system undertakes the function of regulation and controlling to living body. For example, nerve system in human body regulates and controls the particle motion system of human body. Who regulates and controls the volume field motion system of human body? We may suppose that, like nerve system in human body, there exists soul system which consists of soul center system (located in the parts of head and trunk of human body) and soul meridian system. In 


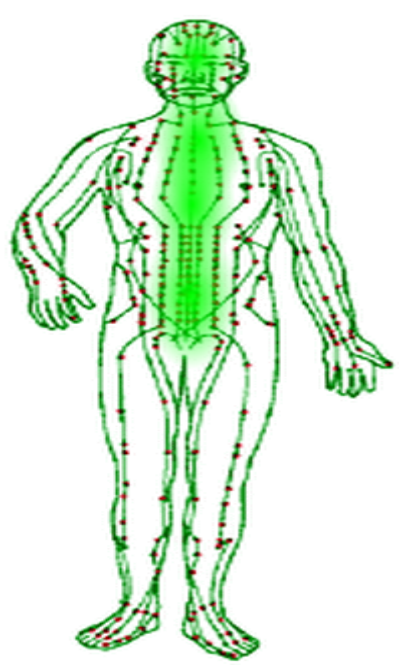

Figure 8. The soul system of human body.

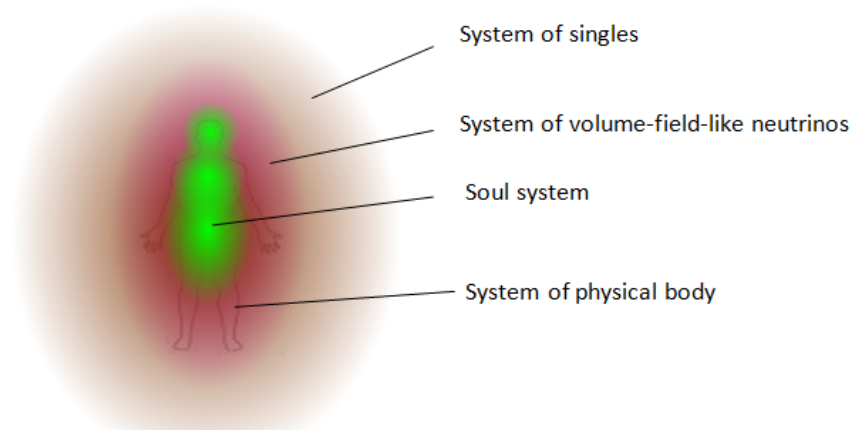

Figure 9. The complex structure of living body.

soul system, there flow some volume-field-like quarks (or "quark chi field" or "strong chi field"), they take part in thinking activities of brain (imaginal thinking) in terms of mind field, serve as energy to organs, and conduct life information along meridian system. Thus, intelligent system of living body is a kind of complex intelligent system which includes nerve system and soul system, nerve system is a kind of particle motion system, soul system is a kind of volume field motion system. Since volume-field-like quarks make strong interaction between them, they destroy the particle structure of soul system, therefore soul system exists in the form of invisibility (Figure 8).

\section{The complex structure of living body}

Through the above discussion we know that, living body is a complex structure system which consists of particle motion system and volume field motion system. The volume field motion system of living body consists of the system of biomacromolecular backbones, soul system (including soul center system and soul meridian system), and the system of volume-field-like neutrinos around all volume fields which carry flavor. The particle motion system of living body consists of the system beyond biomacromolecular backbones and the system of one-in-ones (or singles) around all particles which carry rest mass (Figure 9).
The symmetry principle of particle motion system of non-living body and volume field motion system of living body

The material structure levels of particle motion system of non-living body and the material structure levels of volume field motion system of living body are symmetric

The structure levels of particle motion system of non-living body from small to large are in turn: particle-like fundamental body (including atomic nucleus and extranuclear electron), atom, molecule, molecular polymer, heavenly body, planetary system, stellar system, star cluster, secondary star cluster, tertiary star cluster, and galaxy. The above structure levels of particle motion system of non-living body indicate that, the basic structure unit of particle motion system of non-living body is particle-like fundamental body, on this basis it constructs atom, molecule, molecular polymer, and heavenly body in turn, by heavenly body it constructs planetary system, stellar system, star cluster, secondary star cluster, tertiary star cluster, and galaxy.

The structure levels of volume field motion system of living body from small to large are in turn: volume-field-like fundamental body (including hadronic nucleus and extranuclear quark), hadron, atomic nucleus, biomacromolecular backbone, biomacromolecular polymer (refers to the material part of biomacromolecular backbones, the same below), sub-cellular fraction, cell, tissue, organ, system, and individual organism. The above structure levels of volume field motion system of living body indicate that, the basic structure unit of volume field motion system of living body is volume-field-like fundamental body, on this basis it constructs nucleon, atomic nucleus, biomacromolecular backbone, and biomacromolecular polymer (refers to the material part of biomacromolecular backbones, the same below) in turn, by biomacromolecular polymer it constructs sub-cellular fraction, cell, tissue, organ, system and individual organism (Table 2).

The interaction motion systems of particle motion system of non-living body and the interaction motion systems of volume field motion system of living body are symmetric

Electromagnetic interaction and gravitational interaction are the dominate interactions of particle motion system of non-living body. The dominate interaction of the structure levels bellow molecular polymer (including molecular polymer) is electromagnetic interaction, so molecular polymer (including metal conductor and semiconductor), molecule, and atom are three motion systems of electromagnetic interaction, in these systems atomic nuclei and extranuclear electrons take displacement motion under electromagnetic interaction. The dominate interaction of the structure levels above heavenly body (including heavenly body) is gravitational interaction, so heavenly body, planetary system, stellar system, star cluster, secondary star cluster, tertiary star cluster, and galaxy are all motion systems of gravitational interaction, in these systems heavenly bodies and the fundamental body one-in-one (or singles) which are around heavenly bodies take displacement motion under gravitational interaction.

Similarly, strong interaction and weak interaction are the dominate interactions of volume field motion system of living body. The dominate interaction of the structure levels below biomacromolecular backbone (including bio macromolecular backbone) is strong interaction, so bio macromolecular backbone, atomic nucleus, and hadron are three motion systems of strong interaction, in these systems hadronic nuclei and extra nuclear volume-field-like quarks take volume motion under 
Table 2. The symmetry of material structure levels and the symmetry of interaction motion systems.

\begin{tabular}{|c|c|c|c|c|c|c|c|c|}
\hline motion system & \multicolumn{7}{|c|}{ structure levels } & dominant interaction \\
\hline particle motion system & particle-like fundamental body & atom & \multicolumn{2}{|c|}{ molecule } & \multicolumn{3}{|c|}{ molecular polymer } & electromagnetic interaction \\
\hline $\begin{array}{l}\text { volume field motion } \\
\text { system }\end{array}$ & $\begin{array}{l}\text { volume-field-like fundamental } \\
\text { body }\end{array}$ & hadron & \multicolumn{2}{|l|}{ atomic nucleus } & \multicolumn{3}{|c|}{ biomacromolecular backbone } & strong interaction \\
\hline motion system & \multicolumn{7}{|c|}{ structure levels } & dominant interaction \\
\hline particle motion system & heavenly body & planetary system & stellar system & star cluster & $\begin{array}{l}\text { secondary } \\
\text { star cluster }\end{array}$ & $\begin{array}{l}\text { tertiary star } \\
\text { cluster }\end{array}$ & galaxy & gravitational interaction \\
\hline $\begin{array}{l}\text { volume field motion } \\
\text { system }\end{array}$ & $\begin{array}{l}\text { biomacromolecular polymer } \\
\text { (the part of biomacromolecular } \\
\text { backbones, the same below) }\end{array}$ & $\begin{array}{l}\text { sub-cellular } \\
\text { fraction }\end{array}$ & cell & tissue & organ & system & $\begin{array}{c}\text { individual } \\
\text { organism }\end{array}$ & weak interaction \\
\hline
\end{tabular}

strong interaction. The dominate interaction of the structure levels above bio macromolecular polymer (including bio macromolecular polymer) is weak interaction, so bio macromolecular polymer (refers to the part of bio macromolecular backbones, the same bellow), subcellular fraction, cell, tissue, organ, system, and individual organism are all motion systems of weak interaction, in these systems bio macromolecular polymers and the fundamental body volume-fieldlike neutrinos which are around bio macromolecular polymers take volume motion under weak interaction (Table 2).

\section{References}

1. Thomas Nagel (2012). Mind and Cosmos: Why the Materialist Neo-Darlwinian Conception of Nature is Almost Certainly False. Oxford University Press. pp. 4-5. ISBN 9780199919758.

2. Peter Godfrey-Smith (2013). Philosophy of Biology. Princeton University Press. p. 16. ISBN 9781400850440.

3. Richard H. Jones (2000). "Clarification of terminology". Reductionism: Analysis and the Fullness of Reality. Bucknell University Press. pp. 19 ff. ISBN 9780838754399.

4. Bechtel, William and Robert C. Richardson (1998). Vitalism. In E. Craig (Ed). Routledge Encyclopedia of Philosophy. London: Routledge.

5. Braude, Stephen E. (2002). ESP and Psychokinesis: A Philosophical Examination (Rev. ed.). Parkland, Fla.: Brown Walker Press. P. 21. ISBN 1-58112-407-4.
6. Greene, B. R. (1999). The Elegant Universe. Vintage book. ISBN 978-7-5357-3270-5

7. Chodos, A. Jaffe, R. L. Johnson, K. Thom, C. B. Weisskopf, V. F. (1974) A new extended model of hadrons. Phys Rev 9.

8. Greenberg OW, Zwanziger D (1966) Saturation in triplet model of hadrons. Phys. Rev. 150 .

9. Blatt JM, Weisskopf VF (1952) Theoretical Nuclear Physics. John Wiley \& Sons. P. 94-97.ISBN 0-486-66827-4.

10. Reid RV (1968) Local phenomenological nucleon-nucleon potentials. Annals of Physics 50: 411-448.

11. Muta T (2009) Foundation of quantum chromodynamics: an introduction to perturbative methods in gauge theories (3rd ed.) World Scientific. ISBN 978-981-279-353-9.

12. Smilga A (2001) Lecture on quantum chromodynamics. World scientific. ISBN 978981-02 4331-9.

13. Gell-Mann M (1964) A schematic model of baryons and mesons. Phys Lett 8.

14. Zweig G (1964). An SU(3) model for strong interaction symmetry and its breaking Developments In The Quark Theory Of Hadrons. Vol. 1.

15. Greenberg OW (1964) Spin and unitary spin independence in a paraquark model of baryons and mesons. Phys Rev Lett 13.

16. Penrose R (2005). The road to reality. Vintage book. ISBN 978-00994- 40680.

Copyright: (C2016 Liu R. This is an open-access article distributed under the terms of the Creative Commons Attribution License, which permits unrestricted use, distribution, and reproduction in any medium, provided the original author and source are credited. 